\title{
Topology Control with Radio Interference Detection in Wireless Sensor Network
}

\author{
D. Archana \\ M. Phil Research Scholar \\ Department of Computer Science and Applications \\ Sri Chandrasekhandra Saraswathi Vishwa \\ Mahavidyala University, Kancheepuram
}

\author{
S. Prakasam, Ph.D. \\ Head and Associate Professor \\ Department of Computer Science and Applications \\ Sri Chandrasekhandra Saraswathi Vishwa \\ Mahavidyala University, Kancheepuram
}

\begin{abstract}
Wireless sensor network is a collection of small nodes with sensing environment that will make communication between nodes. Collision free communication in wireless network is performed by medium access control. Energy conservation is important in wireless sensor network that will be performed by topology control. Topology control will route the node with reduce energy and increasing network capacity. While transmission possibility of interference will occur to prevent it from interference a new approach of topology control with radio interference detection is proposed in this paper. This will provide high rate of data transfer without interference for real time communication.
\end{abstract}

\section{Keywords}

Wireless Sensor Networks, Communication, Medium access control, Radio Interference Detection, Topology control.

\section{INTRODUCTION}

Wireless sensor network is a collection of homogeneous and heterogeneous nodes with a capability to interchange the information between sensor nodes using medium access control layer [1].Wireless sensor network have attended and un attended sensor node in large scale, highly distributed in small size based on low power design [2], [3]. Medium access control channel provide a communication between transmitter and receiver to control the collision define a rule for orderly access to the shared medium using access control protocol. Mac use both CSMA and TDMA scheduling. TDMA is one of the conflict free protocols to ensure that a collision can never occur in MAC protocol. TDMA uses topology information to know about neighbour and interference node between sensor nodes. Time slots are scheduled to access in a way that no two nodes collide with each other at the same time using same channel. TDMA gives more importance for accuracy of information and synchronized [4], [5].

Wireless or wired network consist of too many nodes in with a very dense network may cause the problem of collision and difficult to choose a path using a routing protocol. In this situation topology is able to communicate with which other nodes. Sensor nodes are aggregated and transfer the data to the base station that will transmit to the user. Topology control lies on node activity or link activity for communication. Node activity makes node turn on when it is needed and makes off when it is not need. Similarly link is active when it is needed otherwise off [6].

In this section discuss how to route the node with detecting interference as a runtime with a multihop in wireless sensor network. Topology control will reduce power consumption, detention interference, collision and retransmission consequently. It also extends network life time with minimum interference. Radio interference detection is helpful to identify the relation among nodes to communicate with collision free.

\section{RELATED WORK}

Wireless sensor network has a various application such as habitat monitoring [7], Health monitoring, Preventive equipment Maintenance, Military Application. In this application high data rate communication is possible with low energy [8].

Wireless mesh network is focussed on various different applications, so it performance improved with topology control with multichannel and multi radio facilities. It gives importance to power control, multiallocation, routing [9].

Medium access and packet scheduling algorithm must select and transmit packets in accordance with QOS requirements Distributed Dynamic Priority scheduling provide packet transmission in multihop wireless network with high fraction of Quality of Service [10].

DRAND is a fully version of RAND where TDMA distributed scheduling is implemented. TDMA use time slot assignment, so can deliver under high contention. Time is slotted to each node with no overlapping at same time. To avoid conflict colours the node but it is suitable for only within two hop neighbourhood. It is also include in ZMAC, FDMA, CDMA protocol also [11].

In real time conflict free query scheduling adopts a variant of node/link scheduling called query scheduling. In this algorithm time slot are assigned for transmission avoiding collision [12].

In real time transmission scheduling based on wireless hart network is centralized network. Edge colouring, branch bound technique and conflict aware least laxity first based algorithm prohibits simultaneous packet transmission within same channel. This network is limited for a realistic interference [13].

Dynamic Conflict free query Scheduling can efficiently adapt to changes in workloads by exploiting explicit query information provided by the query service [14]

Data flow communication without interference scheduling is real time data flow with the flow planner and flow scheduler by overlapping the transmission of multiple flow instances concurrently [15].

The comparatives table for various techniques used in data transmission and detecting interference is given in table 1 . 
Table. 1 Comparative Table

\begin{tabular}{|c|c|c|c|c|}
\hline Name & Architecture & Algorithm & Function & Draw Back \\
\hline $\begin{array}{l}\text { Radio } \\
\text { Detection }\end{array}$ & $\begin{array}{l}\text { TDMA } \\
\text { Design }\end{array}$ & HD-ND Detection & $\begin{array}{l}\text { i)HD-ND Detection } \\
\text { ii)information sharing } \\
\text { iii)interference } \\
\text { calculation }\end{array}$ & $\begin{array}{l}\text { Unable to perform in large } \\
\text { scale sensor network }\end{array}$ \\
\hline $\begin{array}{l}\text { Real Time Transmission } \\
\text { Scheduling Based On } \\
\text { Wireless hart Network }\end{array}$ & $\begin{array}{l}\text { Centralized TDMA } \\
\text { network management }\end{array}$ & $\begin{array}{l}\text { i)Optimal solution } \\
\text { based on Branch and } \\
\text { bound technique } \\
\text { ii)Heuristic based } \\
\text { Algorithm }\end{array}$ & $\begin{array}{l}\text { Graph edge colouring to } \\
\text { prohibits simultaneous } \\
\text { packet transmission } \\
\text { within same channel }\end{array}$ & $\begin{array}{l}\text { Difficulties in finding } \\
\text { unrealistic interference }\end{array}$ \\
\hline $\begin{array}{l}\text { Drand: Distributed } \\
\text { Randomized Tdma } \\
\text { Scheduling For Wireless } \\
\text { Ad-Hoc Network }\end{array}$ & TDMA Based Design & $\begin{array}{l}\text { Slot Assignment } \\
\text { Algorithm }\end{array}$ & $\begin{array}{l}\text { Finding time slot for } \\
\text { each node in a graph and } \\
\text { assign conflict free nodes } \\
\text { within two hop } \\
\text { neighbourhood }\end{array}$ & $\begin{array}{l}\text { Packet losses when } \\
\text { communication above two } \\
\text { hop and communication } \\
\text { failures does not able to } \\
\text { capture. }\end{array}$ \\
\hline
\end{tabular}

\subsection{Radio Interference Detection Method}

Radio interference detection is real runtime to detect interference among the nodes. The detection can be performed on contention based protocols and TDMA protocols. Radio interference detects relationship among nodes that make a collision free data transmission. Based on signal strength identifies the interference. In this weak link can be possibility of delay deliver while strong link have $100 \%$ packet delivery. In this HD-ND detection sequence to detect the interference node among cluster of node.

RID interference detection is follows in three stages
i) HD-ND detection
ii) Information sharing
iii) Interference calculation.

RID method is used to transmit high power detection (HD packet) and immediately following normal power detection (ND packet) this is called an HD-ND packet detection sequence. The receiver uses HD-ND detection sequence to find interference strength nears to the transmitter side. In HD contain the transmitter ID; from which receiver can be able to find which transmitter, the following ND packet comes. The receiver calculates possible interference caused by the transmitter by sensing the power level of the transmitter's ND packets receives. When Multiple HD-ND detection sequence receives from different transmitter may overlap and disturbance among these detection sequences is detected through the addition rule. The rule is that whether the power level is low during each time period. The receiver mark disturbed HD-ND detection as invalid. Such invalid HD-ND detection node is maintained in the table called Interference-In Table.

After the HD-ND detection follows the information sharing. In this stage finding the hidden terminal node is done. Each node is choosing to use a high sending power to know about interference among its neighbourhood. Normal sending power is also transmit interference among multiple hops. With this information when receiver receives the packet it builds two other tables.
Interference -Out table: this table give information of potential interference for the receiver node.

Interference-HTP table: This table is to find hidden node from the receiver when one of its neighbour is receiving the packet.

Redundancy of nodes is also checked while entering the node in table. This information is enough for finding interference. This information is used for calculating the interference to find all the collision can be occur [16].

\section{PROPOSED SYSTEM}

The proposed system is discussed about how to make transmission of data by selecting a route and also detecting the interference. In Wireless sensor network nodes are very large so it is difficult to find a path in which no interference node can be involved while transmission is takes place. This method will utilize of finding an efficient path using topology control and also detected the interference with the method of radio interference detection. The radio interference will provide accuracy in finding the hidden node in multiple hops.

\subsection{Architecture of Topology Control in Wireless Network}

In wired or wireless network routing is required for transmission. There are various routing algorithm which forward the packet to the destination [17], [18]. For example when node $\mathrm{A}$ wants to send a message to node $\mathrm{B}$, it uses the routing protocol for find a route. This protocol start to discover the route for sending a message with possible hop in the network if no route is found that will make delay in transmission. The routing layer is responsible for transmitting the packets to destination while passing to many intermediate nodes.

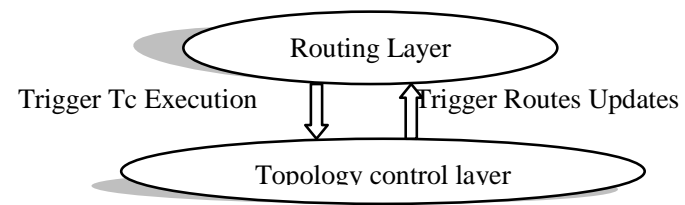

Figure.1 Relation between Routing layer and Topology Control 
The routing protocol performance is improved by using a topology control which create and maintain the list of neighbour node at the time of routing (see Figure 1). According to the route for a new node trigger routes updates the neighbour list for a new node. The list of neighbour node will enter or leave the list according to the change of route for the node. The list also uses triggers Tc execution for break of nodes occurs during transmission.

The medium access control layer is schedule the node to access the shared channel in wireless sensor network (see Figure 2). In medium access control layer various protocols such as CDMA, TDMA, and Z-Mac is designed for conflict free protocol. Among these TDMA is one of the efficient distributed protocols for data transmission [6].

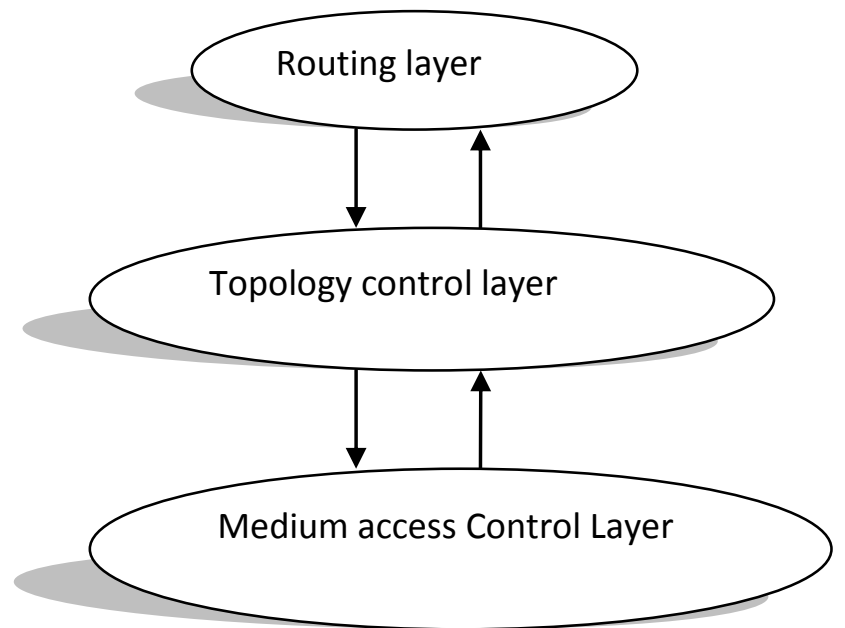

Figure. 2 Relationship between Routing layer, Topology Control and Medium Access control layer

\subsection{Two Phases}

In this system two phases operation are to be performed while data is transmitted. This method will conserve the energy and also provide conflict free transmission with detecting the interference node.

Phase I: This will select a route for transmitting the data using topology control.

Phase II: This will evaluate the interference node with RID

\subsubsection{Phase I}

Topology control is a coordinating node in wireless sensor network which take a decision that which node is in range of transmission with utilizing reduced energy. This method is efficient for energy conservation and increasing network capacity. The communication is performed with short and multipath between the sender and destination. The topology use short path instead of long path in communication due to use of high energy in long path. The transmissions use the short edges with multihop path for the long distance node. This will utilize minimum energy. The main aim of topology control is to find the short path and conserve the energy for transmission. Topology control can be classified as centralized and distributed techniques.

In wireless sensor network transmission distributed topology control is suitable. In this system collection of nodes is represent as graph. A graph $\mathrm{G}=(\mathrm{V}, \mathrm{E})$ where $\mathrm{V}$ is set of nodes and $\mathrm{E}$ is set of edges. An edge $\mathrm{e}=(\mathrm{u}, \mathrm{v})$ exist if only if $\mathrm{u}$ and $\mathrm{v}$ are in $\mathrm{V}$ and $\mathrm{u}$ and $\mathrm{v}$ can hear each other. These graphs form a cluster of nodes based on these formula.

$$
\text { Graph }=(\mathrm{V}, \mathrm{E})
$$

Construct cluster $\mathrm{C}=1 / 2 \mathrm{~V}$

$\forall \mathrm{v} \in \mathrm{V}-\mathrm{C}: \exists c \in C:(V, C) \in E$

$\forall c 1, c 2 \in C:(c 1 . c 2) \notin \mathrm{E}$

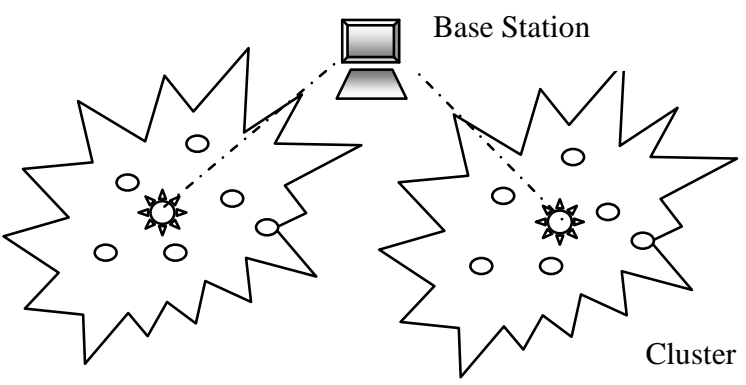

\section{Cluster node Cluster head}

\section{Figure. 3 cluster head and node in wireless sensor network}

Clustering makes partition of nodes in to groups .each group have nodes which are independent among them, no redundancy node in the group (see Figure 3 ). So each group contain different nodes. Groups can have cluster heads all nodes in a cluster are direct neighbour of their cluster head. Cluster head is a dominating set; it should be separated from each other as independent set.

In this cluster have cluster head which is transmit the message to the base station from base station to the receiver is reached. There are several algorithms to implement clustering method [19], [20]. The leader election algorithm is executed in each cluster and cluster nodes elect one of them as the cluster head. The election is based on criteria such as available energy, communication quality and soon. Periodic change of Cluster head is takes place. The cluster head also perform other tasks such as coordinating sensor node sleeping times, aggregating the sensed data provided by the cluster nodes and soon [21].

Message routing is then performed on the basis of a two level hierarchy: the message originated at source cluster node is transmit to the cluster head, which decides whether to forward the message to another cluster head (inter cluster communication) or to deliver the message directly to the destination (intra cluster communication).

Topology control is not itself detecting the interference; it is possible to reduce the minimum interference so we include the RID detection method to detect the interference [22]. At final this phase is up to aggregate the data transmit to the sink but while selecting the intermediate node for transmission there will be possibility of interference node. The interference node is detected in the phase II.

\subsubsection{Phase II:}

In this phase radio interference detection method is used to detect the interference. The radio is competent to detect the interference in this method TDMA is used for $100 \%$ packet delivery with using shared channel at different time to avoid collision. TDMA is perform efficient transmission at the time of heavy load .RID is also suitable for contention based CSMA with back off algorithm to avoid collision .RID can maintain $100 \%$ packet delivery ratio when comparing with other method. RID is able to make collision free time slot 
scheduling. This is runtime detection of interference by knowing relation among nodes.

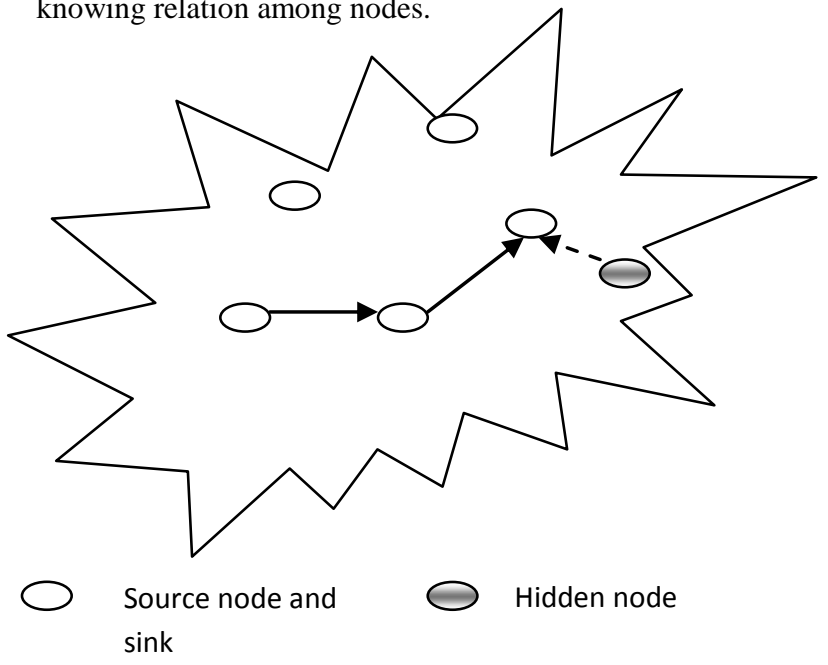

Figure. 4 Hidden node problems

In wireless sensor network there is a problem of hidden node interference this is detected using receiver signal strengths (see Figure 4). It also solves the problem of exposed node detection.

\section{ADVANTAGES OF PROPOSED SYSTEM}

In MAC layer without knowing interference node have drop packets, retransmission of lost packets will occur. To avoid such collision Distributed TDMA-RID will a detect all collision that could happen in the system. TDMA scheduling assign time slots by using knowledge of communication range using shared medium channel. With TDMA packet delivery ratio is increased. To achieve more RID method is used. Interference_In, Interference_Out, Interference_HTP tables are used to achieve collision free scheduling. So with this Mac layer will minimize the packet loss and retransmission function. Acknowledgement is send from the receiver that the packet is received reliable hop by hop communication. It detect all potential interference in communication range and schedule those nodes to transmits in different time slots and avoid collisions when load increases.

Energy consumption also decreases in this system, so more energy is utilize for delivering the packet. Sensor nodes have battery power consumption. While transmission power is wasted for finding the route and transmitting, so with topology control is reconfigurable and efficient schedule with low power MAC Protocol. Topology control previously find the path and schedule the node for transmission at that time node does not use the power it is in sleep mode. Processing will not make use of high energy. So more energy is utilized for communication. Dynamic schedule adapt to current traffic load switch node off ensure that neighbouring nodes turn on simultaneously to allow packet exchange in wireless sensor nodes.

Simultaneous performance of detecting and routing will be consuming energy in the wireless sensor network. Competent data transmission will take place with collision free scheduling.

i) Low power and overlap transmission with topology control.

ii) Distributed TDMA -RID will make $100 \%$ of packet delivery iii) Reduce the number transmitted message to the perform data aggregation sink.

\section{CONCLUSION}

This proposed system designed for real time flow of data in wireless sensor network. Topology control reduces energy consumption and increase network capacities with radio interference detection identify hidden nodes and interference node in the network. This will make efficient way of transmitting the data in wireless sensor network. In future research area is to evaluate, expand this method and detect the congestion and traffic problem while transmission.

\section{ACKNOWLEDGEMENT}

Author express thanks to their relatives and friends for encourage them in good manner for complete this work.

\section{REFERENCES}

[1] Alec Woo, David E Culler, Transmission Control Scheme For Media Access In Sensor Network.

[2] Debroh Estrin, Ramesh Govindan, John Heidemann, Sathish Kumar, Next Century Challenges: Scalable Coordination in Sensor Networks.

[3] I.F.Akyildiz,W.Su,Y.Sankarasubramanian,E.Cayirci, Wireless Sensor Networks: a survey ,March 2002, vol ,pp393-422.

[4] Alberto Leon Garcia, Indra widjaja Communication Networks, Fundamental Concept and Key Architecture, McGraw hill Publication.

[5] Ilker Demirkol, cem Ersoy, Fatih Alagoz,MAC protocols for wireless sensor network a survey.

[6] P Santi, Topology Control in wireless Ad Hoc and Sensor Network, John Wiley \& Sons, Ltd, New York, 2005.

[7] Alan Mainwaring, Joseph polastre, Robert Szewczyk, David culler, John Anderson, Wireless Sensor Networks for Habitat Monitoring.

[8] Lizhi Charlie Zhong, Jan Rabaey, Chun Long Guo, Rahul Shah, Data Link Layer Design for Wireless Networks.

[9] Jatinder Singh saint and Rajan Kumar, July 2013, Wireless Mesh Networks Having Topology Control in Multi-Channel, Multi-Radio.

[10] V.Kanodia, C.Li, A.Sabharwal, B.Sadeghi, E.Knightly, Distributed Multihop Scheduling and medium access with delay and throughput constraints.

[11] Injong Rhee, Ajit Warrier, jeongki min, Lisong xu 2006 DRAND: Distributed Randomized TDMA Scheduling for Wireless AdHoc Networks proc ACM MobiHoc.

[12] S.Jeevitha and S.Kaleeswari, 2013 Optimizing the performance of queries using real time conflict free query scheduling in WSN,proc first IEEE Int'1 conf.Research and Development prospectus on Engineering and Technology.

[13] A.Saifullah, Y.Xu, C.Lu and Y.Chen, 2010 Real time scheduling for Wireless hart networks in RTSS.

[14] Pooja Knnadas, S.Joshua Daniel, Energy Efficient conflict Free Query Scheduling for Wireless Sensor Network, volume 1, Issue-5, and June-2012. 
International Journal of Computer Applications (0975 - 8887)

Volume 109 - No. 10, January 2015

[15] S.Jeevitha ,Data flow communication without interference in wireless sensor networks, june 2013.

[16] G.Zhou, T.He, J.A.Stankovic, and T.F.Abdelzaher, 2005 RID: Radio Interference Detection in WSN, proc .IEEE INFOCOM

[17] Prof.K.Manikandan ,Dr.T.Purosothaman ,An Efficient Routing Protocol Design for Distributed wireless Sensor Netowrk volume10-N.4 November 2010.

[18] Sandhya Bansal, Dr. Dimple Juneja, An Analysis of Real Time Routing Protocols for Wireless Sensor NetworkIJEST.
[19] Savitha M,Shantala Devi Patil,A Survey on Clustering Techniques in wireless Sensor Network Volume 4 Special Issue 1 ,April -2014

[20] Mamta ,Various Clustering Techniques in wireless sensor network, Volume 3,Issue 6-2014

[21] Bhawnesh Kumar,Vinit Kumar Sharma,Distance based cluster Head Selection Algorithm for Wireless Sensor Network, Volume 57-No.9 November 2012.

[22] Martin Burkhart, Pascal Von Rickenbach, Roger Wattenhofer, Aaron Zollinger, Does Topology Control Reduce Interference? 